\title{
Expansion microscopy of cellular and bacterial membranes by functionalized ceramides
}

\author{
Markus Sauer ( $\nabla$ m.sauer@uni-wuerzburg.de ) \\ University of Wuerzburg \\ Ralph Götz \\ University of Wuerzburg \\ Tobias Kunz \\ University of Wuerzburg \\ Julian Fink \\ University of Wuerzburg \\ Franziska Solger \\ University of Wuerzburg \\ Jan Schlegel \\ University of Wuerzburg \\ Jürgen Seibel \\ University of Wuerzburg \\ Vera Kozjak-Pavlovic \\ Vera Kozjak-Pavlovic
}

Thomas Rudel ( $\nabla$ thomas.rudel@biozentrum.uni-wuerzburg.de )

Vera Kozjak-Pavlovic

\section{Method Article}

Keywords: super-resolution imaging, expansion microscopy, membranes

Posted Date: December 2nd, 2020

DOl: https://doi.org/10.21203/rs.3.pex-1249/v1

License: (c) (i) This work is licensed under a Creative Commons Attribution 4.0 International License.

Read Full License 


\section{Abstract}

Due to the lack of primary amino groups, lipids cannot be fixed by formaldehyde, glutaraldehyde and other chemical fixatives nor expanded using expansion microscopy (ExM). We present a protocol that uses unnatural short-chain azide- and amino-functionalized ceramides, which upon incorporation into cellular and bacterial membranes can be labeled by click chemistry and linked into hydrogels, followed by $4 \mathrm{x}$ to $10 \mathrm{x}$ expansion.

\section{Introduction}

Expansion microscopy (ExM) enables super-resolution imaging on standard fluorescence microscopes. By linking a protein of interest into a dense, cross-linked network of a swellable polyelectrolyte hydrogel, biological specimens can be physically expanded allowing $\sim 70 \mathrm{~nm}$ lateral resolution by confocal laser scanning microscopy. Since its introduction by Boyden and co-workers in 2015', expansion microscopy (ExM) has shown impressive results including the magnified visualization of pre- or post-expansion labeled proteins and RNAs with fluorescent proteins, antibodies, and oligonucleotides, respectively, in cells, tissues, and human clinical specimen ${ }^{2}$. In order to be usable for ExM, the molecule of interest has to exhibit amino groups that can react with glutaraldehyde $(\mathrm{GA})^{3}, \mathrm{MA}^{-N H S^{3}}, \mathrm{AcX}^{4}$, or Label- $\mathrm{X}^{5}$ and be linked into the polyelectrolyte hydrogel. The plasma membrane of cells is mainly composed of glycerophospholipids, sphingolipids, and cholesterol. Due to the lack of primary amino groups, these lipids neither can be fixed by formaldehyde, glutaraldehyde and other chemical fixatives nor expanded using available ExM protocols. We used an unnatural short-chain sphingolipid functionalized with an azide and primary amino group to enable fluorescence labeling and chemical fixation as well as linking of the lipid into a swellable hydrogel. Our results demonstrate that the designed unnatural bifunctional sphingolipid is efficiently incorporated into membranes of cells and accumulates in bacterial membranes, which allowed us to investigate the distribution of lipids and interactions with proteins in cellular and bacterial membranes with high spatial resolution.

\section{Reagents}

- $\quad \mathrm{a}-\mathrm{NH}_{2}-\omega-\mathrm{N}_{3}-\mathrm{C}_{6}$-ceramide (homemade)

- $\quad$ 1x PBS (Thermo Fisher, 14190169)

- $\quad 4 \%$ Paraformaldehyde (PFA, Morphisto, 10303.01000)

- Glutaraldehyde 25\% (GA, Sigma Aldrich, G5882)

- $\quad$ Triton X-100 (Thermo Fisher, 28314)

- Hanks' Balanced Salt solution (HBSS, Merck, H6648) 
- $\quad$ Click-IT Alexa Fluor® 488 DIBO alkyne dye (Jena Bioscience, CLK-1278-1)

- Fetal bovine serum (FBS, Sigma-Aldrich, F7524)

- $\quad$ Ammonium persulfate (APS, Sigma, A3678)

- Tetramethylethylenediamine (TEMED, Sigma, T7024)

- Protease K (Thermo Fisher, AM2548)

- $\quad$ Lysozyme (BioChemika, 62970)

- Poly-D-Lysin (PDL, Gibco, A3890401)

- Sodium acrylate (Sigma, 408220)

- $\quad 2.5 \%$ Acrylamide (Sigma, A9926)

- $\quad 0.15 \%$ N,N'-Methylenbisacrylamide (Sigma, A9926)

- $\quad 2 \mathrm{M} \mathrm{NaCl}($ Sigma, S5886)

- $\quad$ DMAA (Sigma, 274135)

- $\quad$ KPS (Sigma, 379824)

- $\quad$ EDTA (Sigma, ED2P)

- Guanidine HCl (Sigma, 50933)

\section{Reagent setup}

For preparation of $4 \mathrm{x}$ monomer solution mix:

- $8.625 \%$ Sodium acrylate (Sigma, 408220)

$-2.5 \%$ acrylamide (Sigma, A9926)

$-0.15 \%$ N, N'-Methylenbisacrylamide (Sigma, A9926)

- $2 \mathrm{M} \mathrm{NaCl}$ (Sigma, S5886)

dissolved in 1x PBS.

For preparation of $10 x$ monomer solution mix: 
$-1 \mathrm{ml}$ of $10 \mathrm{x}$ Monomer solution contains

- 0.267 g DMAA (Sigma, 274135)

- 0.064 g Sodium acrylate (Sigma, 408220)

dissolved in $0.57 \mathrm{~g} \mathrm{ddH}_{2} \mathrm{O}$

The digestion buffer consists of

- $50 \mathrm{mM}$ Tris $\mathrm{pH} 8.0$

- 1 mM EDTA (Sigma, ED2P)

- $0.5 \%$ Triton X-100 (Thermo Fisher, 28314)

- 0.8 M Guanidine HCl (Sigma, 50933)

\section{Equipment}

- 15 mm glass cover slide (VWR, ECN 631-1579)

- Costar 12 Well Cell Culture plate (Corning, 3513)

- Parafilm (Hartenstein, PF10)

- $\quad$ TC dish 150 (Sarstedt, 83.3903)

- $\quad$ Glass chambers (Merck, 734-2055)

\section{Procedure}

Sample preparation

Day 1:

1. Grow cells overnight on a $15 \mathrm{~mm}$ glass cover slide in a 12-well plate in a humidified atmosphere containing $5 \%(\mathrm{v} / \mathrm{v}) \mathrm{CO}_{2}$ at $37^{\circ} \mathrm{C}$.

Day 2:

2. Optional: For sphingolipid ExM of bacteria infect the cells according to standard protocols and follow the protocol after bacterial propagation. 
Note: Ceramides may exhibit toxic effects on some bacterial species.

3. Exchange the media of the cells for fresh media prewarmed to $37^{\circ} \mathrm{C}$

4. Treat the cells with $10 \mu \mathrm{M}$ a- $-\mathrm{NH}_{2}-\omega-\mathrm{N}_{3}-\mathrm{C}_{6}$-ceramide prewarmed to $37^{\circ} \mathrm{C}$ for 1 hour at $37^{\circ} \mathrm{C}$ in the exchanged medium

5. Wash the sample with $1 \times$ PBS

6. Fix the cells using $4 \%$ PFA and $0.1 \%$ GA for 10 minutes

7. Wash the sample 3 times with $1 \times$ PBS

\section{Click-reaction}

8. Permeabilize the sample for 15 minutes in $0.2 \%$ Triton $\mathrm{X}-100$ in $1 \mathrm{x}$ PBS

9. Wash the sample 3 times with 1 xPBS

10. Exchange the PBS for $1 x$ HBSS prewarmed to $37^{\circ} \mathrm{C}$

11. Incubate the sample in $5 \mu \mathrm{M}$ Click-IT Alexa Fluor® 488 DIBO alkyne dye at $37^{\circ} \mathrm{C}$ for 30 minutes. Note: Other functionalized dyes can be used according to preferences.

12. Wash the sample 3 times with $1 x$ PBS

\section{Optional: Immunostaining}

For samples that need additional immunolabelling, e.g. for colocalization of cellular or bacterial proteins, follow the steps a-e before proceeding with step 13.

a. Block the sample with $2 \%$ FBS in $1 \times$ PBS for 1 hour.

b. Incubate the sample in the primary antibody diluted in blocking buffer for 1 hour in a humid chamber

c. Wash the sample 3 times with $1 \times$ PBS

d. Incubate the sample in the corresponding secondary antibody diluted in blocking buffer for 1 hour in a humid chamber

Note: Not all secondary antibodies are suitable for expansion microscopy. Our preferred secondary antibodies are Alexa Fluor 488, CF568, Atto643 and Atto647N.

e. Wash the sample 3 times with $1 \times$ PBS

\section{Expansion Microscopy}


13. Incubate the labelled sample with $0.25 \% \mathrm{GA}$ at RT for 10 minutes.

14. Wash the sample 3 times with $1 \times$ PBS

Note: The monomer solutions for $4 x$ and $10 x$ Expansion are different.

15. For $4 x$ Expansion, add $0.2 \%$ ammonium persulfate (APS) and $0.2 \%$ tetramethylethylenediamine (TEMED) to the $4 x$ monomer solution

16. Immediately pipet $80 \mu \mathrm{l}$ of the mixture on parafilm and turn the labeled $15 \mathrm{~mm}$ glass slide with the cells facing the bottom onto the drop

17. Incubate for 1 hour at RT for gelation

18. For 10x Expansion, degas $1 \mathrm{ml}$ of the 10x monomer solution on ice for 45 minutes with nitrogen.

19. Add $100 \mu \mathrm{KPS}(0.036 \mathrm{~g} / \mathrm{ml})$ to the monomer solution

20. Degas on ice for another 15 minutes with nitrogen

21. Add $4 \mu$ of TEMED

22. Immediately pipet $80 \mu \mathrm{l}$ of the mixture on parafilm and turn the labeled $15 \mathrm{~mm}$ glass slide with the cells facing the bottom onto the drop

23. Incubate for 30 minutes at RT for gelation, followed by 1.5 hours at $37^{\circ} \mathrm{C}$

24. After gelation, remove the gel from the glass cover slide and digest the samples between 3 hours and overnight with $8 \mathrm{U} / \mathrm{ml}$ protease $\mathrm{K}$ in digestion buffer

Note: The cell wall digestion of some bacteria requires additional treatment, e.g. $1 \mathrm{mg} / \mathrm{ml}$ lysozyme in the case of Neisseria gonorrhoeae.

25. Coat glass chambers with PDL overnight at RT.

Day 3:

26. Incubate digested gels in hourly changed $\mathrm{ddH}_{2} \mathrm{O}$ for expansion until expansion is saturated Note: Due to the swelling of the gel, make sure that the fully expanded gel has enough space in the dish used, e.g. TC dish 150.

27. Chop expanded gels in pieces fitting the PDL-coated glass chambers for imaging 


\section{Troubleshooting}

\section{Time Taken}

\section{Anticipated Results}

The described protocol enables nanoscale imaging of cellular and bacterial membranes and differentiation of both layers within double membranes.

\section{References}

1. Chen, F., Tillberg, P.W. \& Boyden, E.S. Optical imaging. Expansion microscopy. Science 347, 543-548 (2015).

2. Wassie, A.T., Zhao, Y. \& Boyden, E.S. Expansion microscopy: principles and uses in biological research. Nature Methods 16, 33-41 (2019).

3. Chozinski, T.J. et al. Expansion microscopy with conventional antibodies and fluorescent proteins. Nature methods 13, 485-488 (2016).

4. Tillberg, P.W. et al. Protein-retention expansion microscopy of cells and tissues labeled using standard fluorescent proteins and antibodies. Nat Biotechno/34, 987-992 (2016).

5. Chen, F. et al. Nanoscale imaging of RNA with expansion microscopy. Nature Methods 13, 679-684 (2016).

This work was supported by the Deutsche Forschungsgemeinschaft (DFG) GRK 2157 to VKP, TR and MS, and DFG FOR 2123 to TR and MS.

\section{Figures}




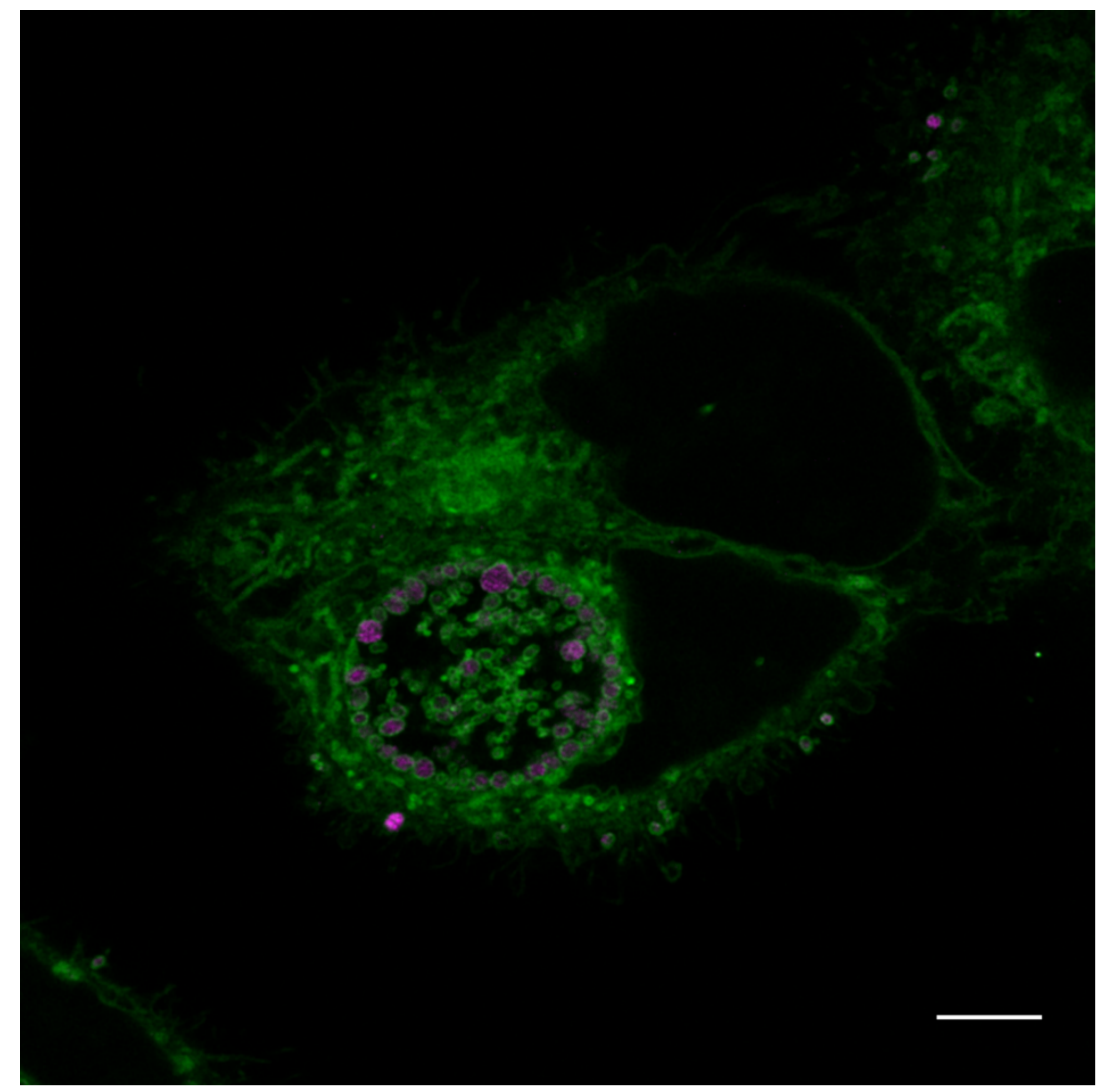

\section{Figure 1}

Sphingolipid ExM enables super-resolution imaging of cellular membranes. Confocal fluorescence image of 4x expanded HeLa229 cells, infected with C. trachomatis, fed with a-NH2-w-N3-C6-ceramide, clicked with DBCO-Alexa Fluor 488 (green) and immunolabeled for chlamydial HSP60 (magenta). Scale bar, 20 $\mu \mathrm{m}$. 


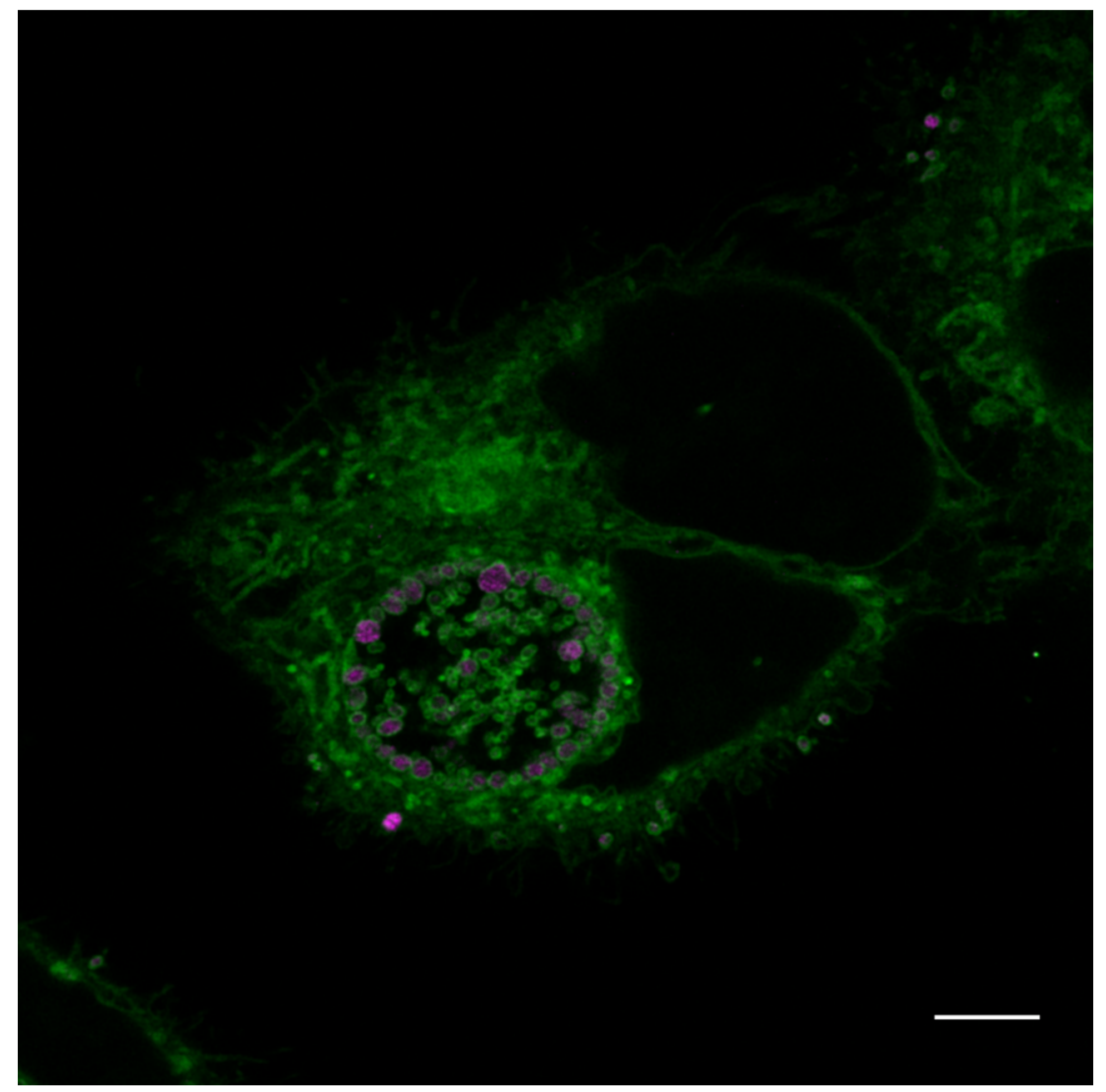

\section{Figure 1}

Sphingolipid ExM enables super-resolution imaging of cellular membranes. Confocal fluorescence image

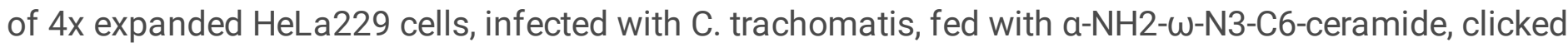
with DBCO-Alexa Fluor 488 (green) and immunolabeled for chlamydial HSP60 (magenta). Scale bar, 20 $\mu \mathrm{m}$. 